\title{
The Effect of Sodium Bicarbonate Administration on the Vasopressor Effect of High-Dose Epinephrine During Cardiopulmonary Resuscitation in Swine
}

\author{
BARRY E. BLESKE, PHARMD, ${ }^{*}$ TED L. RICE, MS, ${ }^{*}$ \\ ERIC W. WARREN, PHARMD, ${ }^{*}$ VINCENT R. DE LAS ALAS, MD, $\dagger$ \\ ALAN R. TAIT, PHD, † PAUL R. KNIGHT, MD, PHD $\ddagger$
}

\begin{abstract}
Sodium bicarbonate is administered during cardiopulmonary resuscitation (CPR) for the treatment of systemic acidemia. However, the effect of administering standard-dose sodium bicarbonate on the vasopressor effect of epinephrine is unknown. This study compared the effects of sodlum blcarbonate or normal saline on the vasopressor effect of epinephrine in 18 pigs. After 10 minutes of unassisted ventricular fibrillation, CPR was started using a pneumatic chest compression device. Two minutes after the start of CPR, sodium bicarbonate $(1 \mathrm{mEq} / \mathrm{kg})$ or normal saline (1 $\mathrm{mL} / \mathrm{kg}$ ) was administered into the right ventricle followed 1 minute later by epinephrine $(0.2 \mathrm{mg} / \mathrm{kg})$. Defibrillation was attempted at 8 minutes of CPR (18 minutes of ventricular fibrillation). Results demonstrated no significant differences in aortic systolic, aortic diastolic, or coronary perfusion pressure (CPP) between the two groups (1 minute after epinephrine, $\mathrm{CPP}$ was $22.6 \pm 13.3 \mathrm{~mm} \mathrm{Hg}$ versus $21.1 \pm 20.7 \mathrm{~mm} \mathrm{Hg}$ for the sodium bicarbonate and normal saline groups, respectively). However, when the data were stratified according to $\mathrm{pH}<7.4$ and $\mathrm{pH}>7.4$, the peak change in CPP was $12.7 \pm 21 \mathrm{~mm} \mathrm{Hg}$ when $\mathrm{pH}<7.4$ and was 5.2 \pm 7.4 when $\mathrm{pH}>7.4(P=.33)$. Resuscitation was also similar between the two groups (two of nine for sodium bicarbonate and one of nine for normal saline). In conclusion, the standard recommended dose of sodium bicarbonate did not alter the vasopressor effect of epinephrine or resuscitation compared with normal saline in this elosed chest model of ventricular fibrillation and CPR. (Am J Emerg Med 1993;11-439-443. Copyright (C) 1993 by W.B. Saunders Company)
\end{abstract}

Systemic acidosis occurs during cardiac arrest and cardiopulmonary resuscitation (CPR). ${ }^{1-3}$ The development of systemic acidosis is the result of decreased oxygen delivery to the tissues consequent to reduced blood flow during CPR. ${ }^{4}$ A potential complication of acidemia during CPR is altered efficacy of adrenergic agents including epinephrine. ${ }^{5-9}$ Epi-

From the *Department of Pharmacy, College of Pharmacy, University of Michigan Hospitals; †Department of Anesthesiology, University of Michigan, Ann Arbor, MI; and fDepartment of Anesthesiology, University at Buffalo, State University of New York, Buffalo, NY.

Manuscript received October 23, 1992; revision accepted February 3, 1993.

Supported by the American College of Clinical PharmacyGlaxo Pharmacotherapy Research Award.

Presented in part at the 13th Annual Meeting of the American College of Clinical Pharmacy, Toronto, Ontario, Canada, August 1992.

Address reprint requests to Dr Bleske, Assistant Professor of Pharmacy, University of Michigan, College of Pharmacy, Ann Arbor, MI 48109-1065.

Key Words: Cardiopulmonary resuscitation, swine, bicarbonates, epinephrine, ventricular fibrillation.

Copyright (c) 1993 by W.B. Saunders Company

$0735-6757 / 93 / 1105-0001 \$ 5.00 / 0$ nephrine has been shown to not only increase coronary perfusion pressure and coronary blood flow, but also to improve survival in CPR studies. ${ }^{10-13}$ Alteration in epinephrine effects caused by acidemia during CPR may have important implications for survival after a cardiac arrest and CPR episode. The current mode of treatment for acidemia observed during CPR is the administration of sodium bicarbonate. ${ }^{14}$ However, it is not known what effect sodium bicarbonate will have on the effect of epinephrine during CPR. Therefore, the purpose of this study was to evaluate the effect of standard-dose sodium bicarbonate $(1 \mathrm{mEq} / \mathrm{kg})$ on the vasopressor effects of epinephrine during CPR after a prolonged period of ventricular fibrillation in a porcine model.

\section{METHODS}

\section{Animal Preparation}

The effect of intravenous epinephrine with and without sodium bicarbonate was evaluated in 18 pigs $(18.8 \pm 4.1 \mathrm{~kg})$ in a randomized and blinded manner. Randomization was achieved by using random numbers with a block design to ensure equal group size. All investigators (except the one investigator who prepared drug) directly involved with the experimental procedure or data analysis were blinded. The study was approved by the University's Unit for Laboratory Animal Medicine. All swine were anesthetized initially with an intramuscular injection of tiletamine $(6 \mathrm{mg} / \mathrm{kg})$, zolazepam $(6 \mathrm{mg} /$ $\mathrm{kg}$ ), and xylazine $(2 \mathrm{mg} / \mathrm{kg}$ ) and were supplemented and maintained with intravenous pentobarbital $(5 \mathrm{mg} / \mathrm{kg})$ as needed. All animals were stabilized for at least 30 minutes after supplemental doses of pentobarbital before beginning any experimental procedures. Each swine was placed on a surgical table with a thermoblanket in a supine position. After the pig was secured, a cuffed endotracheal rube was placed and ventilations started using an Ohio Anesthesia Ventilator (Ohio Medical Products, Madison, WI). The ventilator initially was set at a tidal volume of 10 to $15 \mathrm{~mL} / \mathrm{kg}$ and a respiratory rate of 15 breaths/minute using room air. Blood gases were stabilized for at least 20 minutes before the start of the experimental procedure to an arterial $\mathrm{pH}$ of $7.40 \pm 0.05$ and a partial pressure of carbon dioxide $\left(\mathrm{PCO}_{2}\right)$ of $40 \pm 5 \mathrm{~mm} \mathrm{Hg}$ by altering the respiratory rate or tidal volume if needed. All blood gases were measured using a blood gas analyzer (Radiometer ABL2 Acid-Base Laboratory, Cleveland, $\mathrm{OH}$ ) that was in the same room.

Arterial and venous blood gas samples were obtained before the start of ventricular fibrillation and also at the start of and every minute throughout the CPR period. Arterial samples were obtained from a pig-tailed catheter inserted via the femoral artery with the tail positioned in the aortic arch. Venous samples were obtained through a catheter inserted via the femoral vein with its tip positioned in the inferior vena cava.

Aortic and right atrial pressures were measured and recorded 
throughout the study period. A second pig-tailed catheter placed in the aortic arch was used to obtain arterial pressure measurements, whereas a 6 French catheter with its tip positioned in the right atrium was used to obtain right atrial pressure measurements. The tip of a second 6 French catheter was placed in the right ventricle and used for drug administration. The position of the catheters were determined by evaluating pressure measurements and waveforms with confirmation at the end of the study by necropsy. Pressures were measured with a Gould P23 pressure transducer (after calibration; Gould Inc, Cleveland, $\mathrm{OH}$ ) and recorded along with a singlelead electrocardiogram by a Gould 8 channel RS 3800 graphic recorder (Gould Inc, Cleveland, $\mathrm{OH}$ ). After catheter placement, a 150 $\mathrm{U} / \mathrm{kg}$ heparin bolus was administered intravenously to help maintain catheter patency.

\section{Experimental Protocol}

After obtaining stable baseline measurements, ventricular fibrillation was induced by a $24 \mathrm{~mA}, 60 \mathrm{~Hz}$ electrical current through a pacing wire placed in the right ventricle. After 10 minutes of ventricular fibrillation (without ventilation), CPR was started using a pneumatic chest compression device (Thumper; Michigan Instruments, Grand Rapids, MI) set at a chest compression rate of 80 times/min with a compression duration of 0.5 seconds. At the start of $\mathrm{CPR}$, the compression force was adjusted to produce an initial coronary perfusion pressure gradient (aortic-right atrial middiastolic pressure) during the relaxation phase of approximately $15 \mathrm{~mm} \mathrm{Hg}$. The compression force was not altered once the coronary perfusion pressure gradient had been set. After every fifth compression, diastole was prolonged by 0.5 seconds, and the lungs were inflated to an inspiratory pressure of approximately $10 \mathrm{~cm} \mathrm{H}_{2} \mathrm{O}$ (with $100 \% \mathrm{O}_{2}$ ) by a synchronized, pressure-limited ventilator (Thumper).

After 2 minutes of CPR ( 12 minutes of ventricular fibrillation), sodium bicarbonate $1 \mathrm{mEq} / \mathrm{kg}$ or normal saline $1 \mathrm{~mL} / \mathrm{kg}$ was administered in a randomized and blinded manner followed by a $5 \mathrm{~mL}$ flush of normal saline into the right ventricle. One minute later ( 3 minutes of CPR and 13 minutes of ventricular fibrillation), epinephrine $0.2 \mathrm{mg} / \mathrm{kg}$ was administered followed by a $5-\mathrm{mL}$ flush of normal saline into the right ventricle.

After 8 minutes of CPR (18 minutes of ventricular fibrillation), external defibrillation was attempted with $7 \mathrm{~J} / \mathrm{kg}$ of energy (model 604-A; Mennen/Greatbach Electronics, Inc, Clarence, NY). If the pig remained in ventricular fibrillation, $C P R$ was continued. Further attempts at defibrillation using $10 \mathrm{~J} / \mathrm{kg}$ of energy were made every 90 seconds until ventricular fibrillation was terminated or a total time of 10 minutes had elapsed from the first defibrillation attempt. For this study, the definition of resuscitation was attainment of an organized cardiac rhythm with a systolic blood pressure of $60 \mathrm{~mm} \mathrm{Hg}$ or more for at least 2 minutes within a 12-minute period from the first defibrillation without the use of additional vasopressor support. ${ }^{15}$

\section{Data Analysis}

Aortic and right atrial pressures were determined using the average of five readings per measurement by a blinded investigator. Statistical comparison of the two treatment groups was performed using analysis of variance with a repeated measures design. The relationship between blood gas parameters and myocardial pressures was evaluated by Pearson's correlation coefficient. A $P \leqslant .05$ was considered the critical probability level for all tests. Data are reported as mean and standard deviation.

\section{RESULTS}

A total of 18 pigs were studied $(\mathrm{n}=9$ for the sodium bicarbonate group, $\mathrm{n}=9$ for the normal saline group). Mean pressures over time during CPR are listed in Table 1 for the sodium bicarbonate and normal saline groups. Both groups produced an increase in aortic systolic, aortic diastolic, and coronary perfusion pressures over time with no significant differences between the groups at any point measured. There were no differences in pressures after sodium bicarbonate administration compared with baseline values. Additionally, there was no difference between groups when adjusted for baseline differences. Both groups demonstrated similar resuscitation rates with two of nine pigs surviving in the sodium bicarbonate treatment group and one of nine pigs in the normal saline group.

Mean aortic blood gas values ( $\mathrm{pH}, \mathrm{PCO}_{2}$, and bicarbonate) over time are listed in Table 2. No significant differences between groups were observed in aortic blood gas values before ventricular fibrillation or at the start of CPR. The sodium bicarbonate group demonstrated significant elevations in bicarbonate concentrations. No significant differences were observed for $\mathrm{pH}$, hydrogen ion concentrations, or $\mathrm{PCO}_{2}$ values despite increases in these measurements after sodium bicarbonate administration (ie, $\geqslant 3$ minutes after the start of CPR). However, when these values were adjusted for baseline differences (change from the values obtained 2 minutes after the start of CPR) significant differences were observed between the two groups. Specifically, significant increases were observed at 3,4 , and 5 minutes after the start of CPR for $\mathrm{pH}$ values in the sodium bicarbonate group compared with the normal saline group $(0.075 \pm 0.082,0.106 \pm$ $0.151,0.081 \pm 0.17$ vs $-0.028 \pm 0.037,-0.052 \pm 0.068$, $-0.076 \pm 0.078$, respectively; $P<0.03$ ). In addition significant increases in $\mathrm{PCO}_{2}(\mathrm{~mm} \mathrm{Hg})$ were observed in the sodium bicarbonate group compared with the normal saline

TABLE 1. Mean Pressure (mm Hg) During CPR

\begin{tabular}{|c|c|c|c|c|c|c|c|}
\hline & $2 \mathrm{MIN}^{*}$ & $3 \mathrm{MINT}$ & 4 MIN & 5 MIN & $6 \mathrm{MIN}$ & $7 \mathrm{MIN}$ & $8 \mathrm{MIN}$ \\
\hline \multicolumn{8}{|c|}{ Bicarbonate group } \\
\hline AOS & $39.9 \pm 21.2$ & $38.6 \pm 20$ & $54.1 \pm 36.4$ & $58.2 \pm 31.0$ & $57.6 \pm 29.9$ & $55.7 \pm 29.2$ & $54.8 \pm 29.8$ \\
\hline AOD & $19.9 \pm 7.3$ & $18.9 \pm 6.4$ & $27.8 \pm 15.9$ & $29.1 \pm 12.6$ & $27.9 \pm 11.3$ & $26.4 \pm 11.0$ & $25.7 \pm 10.4$ \\
\hline CPP & $16.6 \pm 7.3$ & $16.0 \perp 5.9$ & $22.6 \pm 13.3$ & $24.3 \pm 11.7$ & $22.7 \pm 10.7$ & $22.1 \pm 10.9$ & $21.1 \pm 11$ \\
\hline \multicolumn{8}{|c|}{ Normal saline group } \\
\hline AOS & $\begin{array}{l}53.2 \pm 44 \\
17.4 \pm 6.1\end{array}$ & $\begin{array}{l}52.7 \pm 41 \\
17.7 \pm 5.8\end{array}$ & $\begin{array}{l}64.7 \pm 64 \\
24.9 \pm 20.1\end{array}$ & $\begin{array}{l}64.3 \pm 61 \\
25.6 \pm 19.5\end{array}$ & $\begin{array}{l}62.8 \pm 54 \\
24.8 \pm 15.7\end{array}$ & $\begin{array}{l}63.1 \pm 55 \\
24.0 \pm 15.7\end{array}$ & $\begin{array}{l}60.6 \pm 54 \\
22.2 \pm 13.7\end{array}$ \\
\hline CPP & $15.0 \pm 6.7$ & $14.8 \pm 6.7$ & $21.1 \pm 20.7$ & $21.8 \pm 20.4$ & $20.5 \pm 16$ & $19.9 \pm 16$ & $18.1 \pm 13.8$ \\
\hline
\end{tabular}

Abbreviations: AOS, aortic systolic pressure; $A O D$, aortic diastolic pressure; CPP, coronary perfusion pressure; MIN, minutes.

* Minutes after the start of cardiopulmonary resuscitation.

† Epinephrine given. 

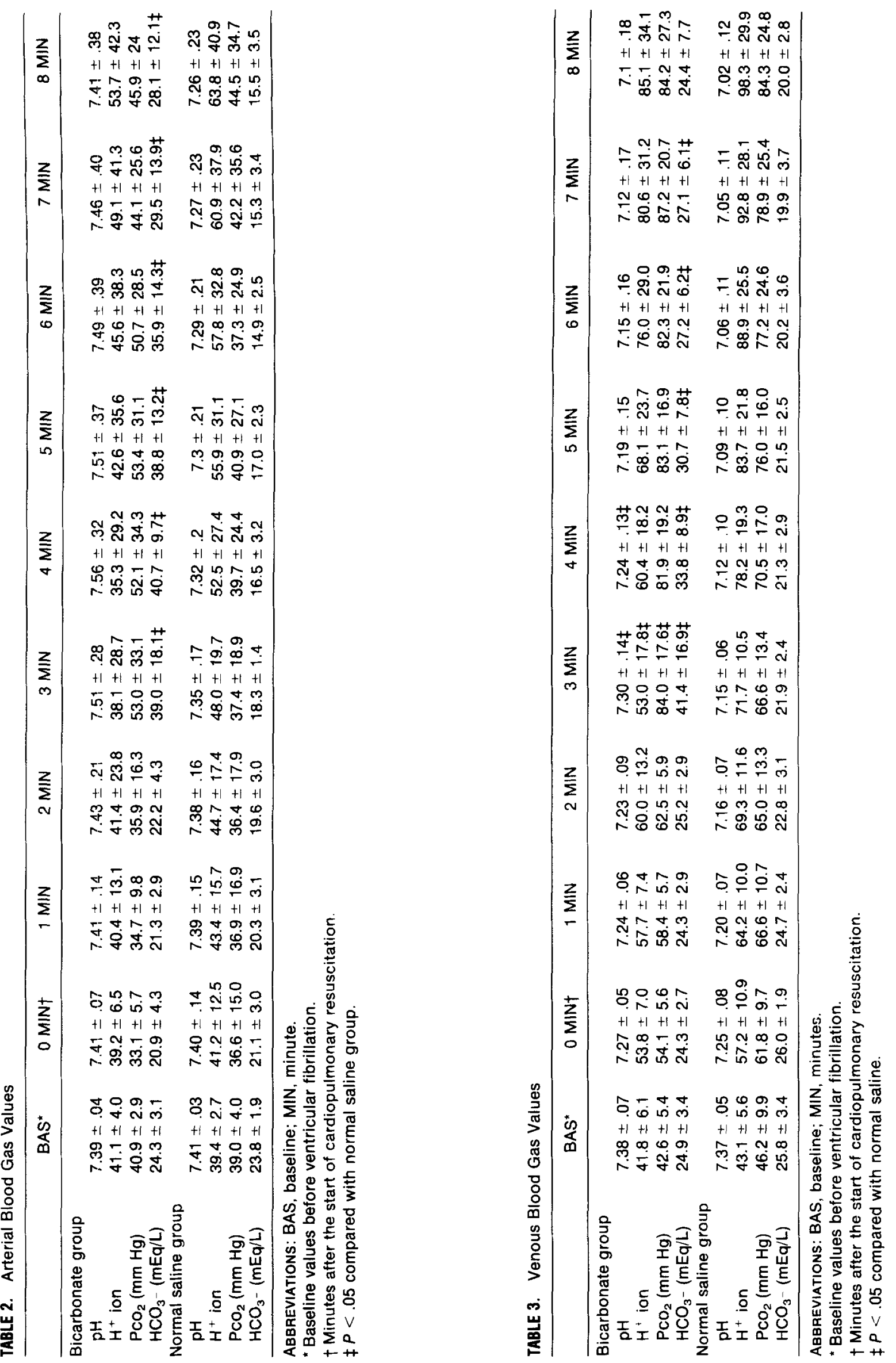
group at $3,4,5$, and 6 minutes of CPR $(18 \pm 19.2,18.1 \pm 17$. $18.4 \pm 15.7,15.8 \pm 13.6$ vs $1 \pm 6.6,3.3 \pm 8.9,4.5 \pm 9.6,1$ \pm 8 , respectively; $P \leqslant .04$ ). Correlation between arterial blood gas values and pressures (adjusted for baseline differences) demonstrated no significant correlation between $\mathrm{pH}$, $\mathrm{PCO}_{2}$, or bicarbonate values and pressure changes.

Mean venous blood gas values $\left(\mathrm{pH}, \mathrm{PCO}_{2}\right.$, and bicarbonate) over time are listed in Table 3. No significant differences between groups were observed in venous blood gas values before ventricular fibrillation or at the start of CPR. However, the sodium bicarbonate group demonstrated significant elevations in $\mathrm{pH}$, hydrogen ion concentrations, $\mathrm{PCO}_{2}$, and bicarbonate measurements after sodium bicarbonate administration (ie, $\geqslant 3$ minutes after the start of CPR).

Correlation between venous blood gas values and pressures showed similar results as the arterial blood gas values for $\mathrm{pH}$ and bicarbonate analysis. However, a weak correlation was observed between $\mathrm{PCO}_{2}$ values and both coronary perfusion pressure and aortic diastolic pressure changes $(r-$ $.42, P=.0001$ and $r=.45, P=.0001$, respectively) showing that as $\mathrm{PCO}_{2}$ increases, aortic diastolic and coronary perfusion pressure increases.

When peak change in coronary perfusion pressure for each individual animal was stratified according to $\mathrm{pH}$ regardless of sodium bicarbonate administration, the results show that when $\mathrm{pH}<7.4(\mathrm{pH}=7.17 \pm 0.14 ; \mathrm{n}=9$; six normal saline and three sodium bicarbonate pigs) peak change in coronary perfusion pressure was $12.7 \pm 21 \mathrm{~mm} \mathrm{Hg}$ versus $5.2 \pm 7.4 \mathrm{~mm} \mathrm{Hg}(P=.33)$ when $\mathrm{pH}>7.4(\mathrm{pH}=7.65 \pm$ $0.16 ; \mathrm{n}=9$; six sodium bicarbonate and three normal saline pigs). When the high and the low values were eliminated, the peak change in coronary perfusion pressure was $7.7 \pm 8.4$ $\mathrm{mm} \mathrm{Hg}$ at $\mathrm{pH}=7.19 \pm 0.1$ and $3.6 \pm 3.1$ at $\mathrm{pH}=7.68 \pm .17$ $(P=.25)$.

\section{DISCUSSION}

The effect of sodium bicarbonate on arterial and venous acid-base status has been well documented; however, the effects of sodium bicarbonate on the pressure response to epinephrine is less well defined. The results from this study demonstrated that there was no difference in the pressures evaluated or in resuscitation after epinephrine administration between the sodium bicarbonate or normal saline groups. Evaluation of arterial and venous blood gases demonstrated elevations in $\mathrm{pH} . \mathrm{PCO}_{2}$, and bicarbonate values after sodium bicarbonate administration compared with normal saline administration, which is consistent with previous studies. ${ }^{16,17}$

In this animal model of ventricular fibrillation and CPR, sodium bicarbonate administration has no effect on the vasopressor response to epinephrine administration. These results are similar to a previous study in swine evaluating sodium bicarbonate $(3 \mathrm{mEq} / \mathrm{kg}$ ) and epinephrine $(0.04 \mathrm{mg} /$ $\mathrm{kg}) .^{18}$ However, there are differences between studies, including the dose of sodium bicarbonate used. the mode of CPR (open chest vs closed chest), and baseline coronary perfusion pressure, which was twice as high as our study (approximately $15 \mathrm{~mm} \mathrm{Hg}$ vs $30 \mathrm{~mm} \mathrm{Hg}$ ). Both studies contain important findings, but of the two studies, our study represents a more clinically applicable model secondary to the type of CPR performed (closed chest model) and lower baseline coronary perfusion pressure. A recent study in humans demonstrated that the baseline coronary perfusion pressure in patients who had return of spontaneous circulation was only $13.4 \pm 8.5 \mathrm{~mm} \mathrm{Hg}$, whereas patients who did not have return of circulation had baseline coronary perfusion pressure of $1.6 \pm 8.5 \mathrm{~mm} \mathrm{Hg} .{ }^{19}$ The elevated coronary perfusion pressure observed in the previous study may bias the results and not be reflective of an actual CPR setting.

Our study also showed, before epinephrine administration, that sodium bicarbonate had no effect on coronary perfusion pressure. This is in contrast to previous studies that show that the administration of sodium bicarbonate (without epinephrine) decreases coronary perfusion pressure in closed chest models of CPR. ${ }^{20.21}$ However, these studies used a larger dose of sodium bicarbonate than this study, which used the standard $1 \mathrm{mEq} / \mathrm{kg}$ dose. These results suggest that when sodium bicarbonate is administered alone it may decrease coronary perfusion pressure in a dosedependent manner in a closed chest model of CPR. The potential mechanism for the decrease in coronary perfusion pressure observed in these studies is thought to be caused by the vasodilatory effect of a hyperosmolar solution. ${ }^{20,21}$

In evaluating pressures after sodium bicarbonate administration. the relationship between blood gas values and pressure changes were evaluated. Overall, there was no significant correlation between blood gas valucs and changc in pressure except for the weak correlation between venous $\mathrm{PCO}_{2}$ and pressure, indicating that as venous $\mathrm{PCO}_{2}$ values increased, pressure increased. However, when only the maximal change in coronary perfusion pressure was evaluated according to $\mathrm{pH}$, there was a trend suggesting that lower the $\mathrm{pH}(<7.4)$ the greater the increase in coronary perfusion pressure. In fact, coronary perfusion pressure tended to be twice as high in the group that had arterial acidemia. A recent study in humans has shown similar results in which patients with a $\mathrm{pH}$ of more than $7.42 \mathrm{had}$ significantly lower coronary perfusion pressure during CPR after epinephrine administration $(0.2 \mathrm{mg} / \mathrm{kg})$ compared with patients with a $\mathrm{pH}$ of less than $7.38(4 \pm 6 \mathrm{~mm} \mathrm{Hg}$ vs $12 \pm 9$ $\mathrm{mm} \mathrm{Hg}, P<.0001$, respectively). ${ }^{22}$ These data suggest that overzealous administration of sodium bicarbonate leading to severe arterial alkalemia may not be beneficial in regards to obtaining optimal coronary perfusion pressure. Further work in this area is required.

Even though pressures increased after epinephrine administration. the overall magnitude of this increase was lower than what has been reported previously with $0.2 \mathrm{mg} / \mathrm{kg}$ dose of epinephrine. ${ }^{23,24}$ The reason for this is not clear because this model is somewhat similar to previous models and epinephrine was administered directly into the right ventricle. One possible explanation for our results is that we had a more severe model of cardiac arrest because of a number of possible reasons (including species of pig used, CPR technique, etc), which resulted in a decreased response to epinephrine.

Our study demonstrated limited survival in both groups. Previous studies have shown higher survival rates after 0.2 $\mathrm{mg} / \mathrm{kg}$ epinephrine therapy. ${ }^{23,24}$ In addition, other studies evaluating sodium bicarbonate therapy demonstrated either improved or similar survival compared with normal saline. ${ }^{18,21,25-27}$ Differences between studies are related to de- 
sign, drug dosages, and most critically, coronary perfusion pressures. When coronary perfusion pressures are high (probably more than $25 \mathrm{~mm} \mathrm{Hg}$ ), most animals will survive regardless of treatment randomization. ${ }^{10-12,25}$ Therefore. evaluating survival in these type of studies is probably limited because coronary perfusion pressure is the major determinant of survival. Because coronary perfusion pressure is critical in animal models of CPR, the major end point for this study was changes in pressure and not survival. The limited survival observed in our study is consistent with the minimal pressure response observed after epinephrine administration, the prolonged CPR period after epinephrine administration, and no additional resuscitation measures (ie, additional epinephrine administration) after defibrillation.

The results from this study must be interpreted with caution because our model represents a prolonged period of ventricular fibrillation and CPR. The results from models using shorter periods of ventricular fibrillation and CPR or models that demonstrate greater response to epinephrine is unknown. Whether or not the dose of epinephrine is important (ie, high dose vs standard dose) in regards to pressure response is also unknown. Additionally, our results may have been different if systemic alkalemia and acidemia had been more extreme or a larger dose of sodium hicarbonate had been used. Using extreme end points may have made detecting a difference between groups easier. Finally, evaluation of energy metabolism and neurological scores after resuscitation may also be beneficial.

In conclusion, this study demonstrated that standard 1 $\mathrm{mEq} / \mathrm{kg}$ dose of sodium bicarbonate had no effect on the vasopressor response to epinephrine compared with normal saline in this closed-chest model of prolonged ventricular fibrillation and CPR. This study also suggests that arterial acidemia may not decrease the vasopressor response to epinephrine and may actually improve response. Overall, it appears that there may be limited reason to administer sodium bicarbonate during ventricular fibrillation and CPR in attempt to improve the vasopressor response to epinephrine.

\section{REFERENCES}

1. Weil MH, Rackow EC, Trevino R, et al: Difference in acidbase state between venous and arterial blood during cardiopulmonary resuscitation. $N$ Engl J Med 1986;315:153-156

2. Fillmore SJ, Shapiro M, Killip T: Serial blood gas studies during cardiopulmonary resuscitation. Ann Intern Med 1970;72: 465-469

3. Adrogue $\mathrm{HJ}$, Rashad N, Gorin AB, et al: Assessing acidbase status in circulatory failure. Differences between arterial and central venous blood. N Engl J Med 1989;320:1312-1316

4. Del Guercio LRM, Feins NR, Cohn JD, et al: Comparison of blood flow during external and internal cardiac massage in man. Circulation 1965:32:171-180 (suppl)

5. Korstanje C, Mathy MJ, van Charldorp K, et al: Influence of respiratory acidosis or alkalosis on pressor responses mediated by alpha-1 and alpha-2 adrenoceptors in pithed normotensive rats. Naunyn Schmiedebergs Arch Pharmacol 1985;330:187-192

6. deHurtado MCC, Argel MI, Cingolani HE: Influence of acid-base alterations on myocardial sensitivity to catecholamines. Naunyn Schmiedebergs Arch Pharmacol 1981;317:219224

7. Grant TL, McGrath JC, O'Brien JW: The influence of blood gases on alpha- 1 and alpha-2 adrenocepto-mediated pressor responses in the pithed rat. Br J Pharmacol 1985;86:69-77
8. Houle DB, Weil MH, Brown EB, et al: Influence of respiratory acidosis on ECG and pressor response to epinephrine, norepinephrine, and metaraminol. Proc Soc Exp Biol Med 1957;94: $561-564$

9. Wildenthal K, Mierzwiak DS, Myers RW, et al: Effects of acute lactic acidosis on left ventricular performance. Am J Physiol 1968;214:1352-1359

10. Kern K, Vorrhees W, Babbs C, et al: Myocardial perfusion pressure during prolonged cardiopulmonary resuscitation predicts 24 hour survival. Crit Care Med 1986;14:334 (abstr)

11. Sanders A, Ogle M, Ewy G: Coronary perfusion pressure during cardiopulmonary resuscitation. Am J Emerg Med 1985; 3:11-14

12. Nieman JT, Criley JM, Rosborough JP, et al: Predictive indices of successful resuscitation after prolonged arrest and experimental cardiopulmonary resuscitation. Ann Emerg Med 1985; 14:521-528

13. Brown CG, Werman WA, Davis EA, et al: The effects of graded doses of epinephrine on regional myocardial blood flow during cardiopulmonary resuscitation in swine. Circulation $1987 ; 75: 491-497$

14. American Heart Association and National Academy of Sciences-National Research Council. Standards and guidelines for cardiopulmonary resuscitation (CPR) and emergency cardiac care (ECC). JAMA 1986;255:2905-2992

15. Bleske BE, Warren EW, Rice TL, et al: Comparison of intravenous and intranasal administration of epinephrine during CPR in a canine model. Ann Emerg Med 1992;21:1125-1130

16. Sanders $A B$, Otto $C W$, Kern $K B$, et al: Acid-base balance in a canine model of cardiac arrest. Ann Emerg Med 1988;17:667671

17. Spivey $W H$, Lathers CM, Malone DR, et al: Comparison of intraosseous, central and peripheral routes of sodium bicarbonate administration during CPR in pigs. Ann Emerg Med 1985;14: 1135-1140

18. Federiuk CS, Sanders AB, Kern KB, et al: The effect of bicarbonate on resuscitation from cardiac arrest. Ann Emerg Med 1991;20:1173-1177

19. Paradis NA, Martin GB, Rivers EP, et al: Coronary perfusion pressure and the return of spontaneous circulation in human cardiopulmonary resuscitation. JAMA 1990;263:1106-1113

20. Kette F, Wei MH, Gazmuri RJ: Buffer solutions may compromise cardiac resuscitation by reducing coronary perfusion pressure. JAMA 1991;266:2121-2126

21. Gazmuri RJ, vonPlanta $M$, Weil $M H$, et al: Cardiac effects of carbon dioxide-consuming and carbon dioxide-generating buffers during cardiopulmonary resuscitation. J Am Coll Cardiol $1990 ; 15: 482-490$

22. Paradis NA, Goetting MG, Rivers EP, et al: The effect of $p H$ on the change in coronary perfusion pressure after epinephrine during CPR in human beings. Ann Emerg Med 1990:19:457 (abstr)

23. Brown CG, Katz SE, Werman HA, et al: The effect of epinephrine versus methoxamine on regional myocardial blood flow and defibrillation rates following a prolonged cardiorespiratory arrest in a swine model. Am J Emerg Med 1987:5:362-369

24. Brown CG, Taylor RB, Werman HA, et al: Myocardial oxygen delivery/consumption during cardiopulmonary resuscitation: A comparison of epinephrine and phenylephrine. Ann Emerg Med 1988;17:302-308

25. Guerci AD, Chandra N, Johnson E, et al: Failure of sodium bicarbonate to improve resuscitation from ventricular fibrillation in dogs. Circulation 1986;74:IV-75-IV-79 (suppl IV)

26. Sanders AB, Kern KB, Fonken $S$, et al: The role of bicarbonate and fluid loading in improving resuscitation from prolonged cardiac arrest with rapid manual chest compression CPR. Ann Emerg Med 1990;19:1-7

27. Vukmir RB, Bircher NG, Safar P, et al: Effect of sodium bicarbonate on survival in a canine 15-minute cardiac arrest model. Ann Emerg Med 1991;20:491 (abstract) 